OPEN ACCESS

Edited by:

Jinjin Li,

Shanghai Jiao Tong University, China

Reviewed by:

Xihua Zou,

Southwest Jiaotong University, China Yu Liu, Institute of Semiconductors (CAS),

China

*Correspondence:

Yali Zhang

ylzhang@uestc.edu.cn

Specialty section:

This article was submitted to

Optics and Photonics,

a section of the journal

Frontiers in Physics

Received: 19 May 2021

Accepted: 15 June 2021

Published: 29 July 2021

Citation:

Zhang Y, Liao S, Wang G, Yang K,

Zhang Z, Zhang S and Liu Y (2021)

Robust Optical Frequency Comb

Generation by Using a Three-Stage

Optical Nonlinear Dynamic.

Front. Phys. 9:711959.

doi: 10.3389/fphy.2021.711959

\section{Robust Optical Frequency Comb Generation by Using a Three-Stage Optical Nonlinear Dynamic}

\author{
Yali Zhang *, Shuxu Liao, Guan Wang, Ke Yang, Zhiyao Zhang, Shangjian Zhang and Yong Liu
}

State Key Laboratory of Electronic Thin Films and Integrated Devices, School of Optoelectronic Science and Engineering, University of Electronic Science and Technology of China, Chengdu, China

In this article, we propose and investigate a novel scheme to generate optical frequency combs (OFCs) by using a three-stage generator, which is based on optical injection-induced dynamics cascaded by subharmonic electro-optic modulation and the four-wave mixing (FWM) effect. A primary seed OFC is rooted from the nonlinear dynamics in the optically injected semiconductor laser, and its performance is improved using a two-stage booster based on subharmonic electro-optic modulation and the FWM effect. The comb spacing can be easily tuned by adjusting that of the primary seed OFC or through electro-optic modulation by the use of subharmonics with different orders. Moreover, it becomes stabilized because the phase relationship between the comb teeth can be fixed in the process of subharmonic electro-optic modulation. Its optical spectrum continues to be broadened in the following FWM process. Finally, robust OFCs with a comb spacing of $4 \mathrm{GHz}$ and a comb teeth number of 23 and a comb spacing of $5 \mathrm{GHz}$ and a comb teeth number of 21 are experimentally demonstrated.

Keywords: optical frequency comb, optical injection, subharmonic electro-optic modulation, four-wave mixing, nonlinear dynamics

\section{INTRODUCTION}

As a special kind of ultrashort optical pulse, optical frequency combs (OFCs) have important applications in various fields, ranging from examining the theory of quantum electro-dynamics [1, 2], space and time variations of fundamental constants [3-5], optical frequency synthesis [6], optical communications [7], and precise optical metrology [8] to optical arbitrary waveform generation [9] and so on. For example, it can realize high-precision measurement of optical frequency. The core of such high-precision optical spectroscopy is due to high frequency stabilities and accuracies of ultranarrow light sources at the desired wavelengths provided by the OFC [10].

Over the past decades, to meet the preference demand on OFCs in various application scenarios, various kinds of generation techniques for OFCs have been proposed, which have demonstrated their own distinct characteristics. These generation techniques can be generally categorized into six main types. The two classical ones are based on mode-locked lasers (MLLs) [11, 12] and on cyclic frequency shift structures $[13,14]$. The four others can all be classified into the microwave photonic domain, namely, based on modulators [15, 16], based on optoelectronic oscillators (OEOs) [17, 18], based on nonlinear optical fibers [19,20], and based on optically injected semiconductor lasers [21, 22]. For OFCs based on modulators, the phase relationship between the comb teeth is fixed due to the known electro-optic effect, the frequency stability is robust, and comb spacing can be flexibly adjusted by changing the frequency of the modulation signal. However, the number of comb teeth is 
A

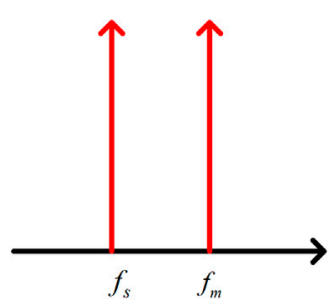

C

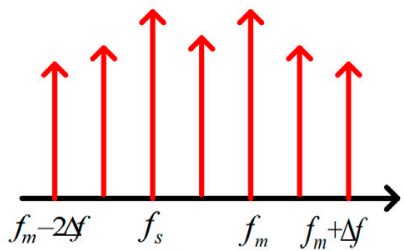

B

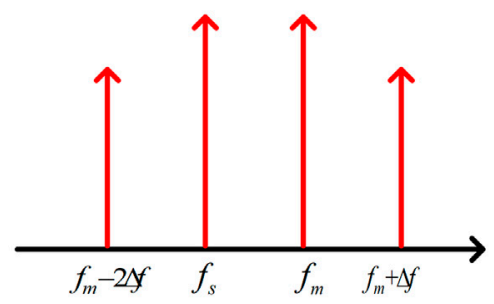

D

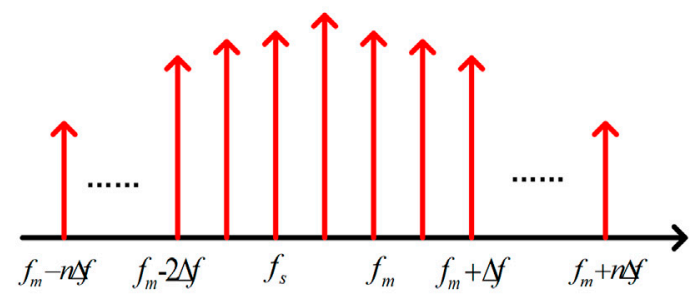

FIGURE 1 | Principle diagram of the proposed generation system of the OFC. Spectra diagram of (A) free-running ML and SL lasers, (B) primary seed OFC, (C) subharmonic-locked OFC, and (D) FMW-broadened OFC.

small and the flatness is poor, both of which are limited by the power of the modulation signal and the bandwidth of the used modulators. The comb spacing of OFCs based on MLLs can also be adjustable, for the wavelength tuning range of MLLs is large and its repetition frequency can be adjusted in a certain range. Yet its flexibility is poor, for the repetition frequency of MLLs can only be tuned in a small range. The OFC based on cyclic frequency shift structures has numbers of comb teeth and good flatness, whereas it has disadvantages such as an unclear phase relationship between comb teeth and high carrier noise. The OFC generated by the self-oscillation in the optoelectronic oscillation loop does not need an external microwave source, which can help avoid the additional phase noise caused by the external microwave source. At the same time, OEOs can provide a stable microwave signal with stable ultralow noise to reduce the phase noise of the generated OFC. However, not only is the system structure of the scheme complex and expensive but also the number of comb teeth is relatively small and the comb spacing cannot be changed due to the limited narrow bandwidth of the electronic filter in the OEO loop. The OFC based on nonlinear effects in optical fibers has numbers of comb teeth, but the flatness is poor. What is worse is that the number of comb teeth cannot be controlled because it is difficult to quantitatively control nonlinear effects. Interestingly, the optical spectrum of the period-one oscillation state induced in the optically injected semiconductor laser has several frequency modes, and the frequency spacing between these modes is strictly equal, which is exactly an embryonic form of OFCs. Therefore, it has the potential to generate OFCs based on optical injection technology. It has been found that the nonlinear period-one dynamic can successfully provide seed OFCs. The process of optical injection in the resonance cavity of the laser can ensure coherence among the comb teeth, and it can realize the flexile adjustment of comb spacing in principle. In addition, the optical injection technology is simple in structure, rich in theoretical analysis, and mature in technology. However, such primary seed OFCs are quite sparse in number and uneven in flatness with respect to comb teeth, and the phase relationship between the comb teeth may become unstable due to the fluctuation noise originating in the resonance cavity of the laser.

In this study, we employ the primary OFC that originated from the process of optical injection as the seed OFC. We propose and demonstrate that improved OFCs with adjustable comb spacing and excellent frequency stability can be obtained with cascaded subharmonic electro-optic modulation and the fourwave mixing (FWM) effect. Finally, robust OFCs with a comb spacing of $4 \mathrm{GHz}$ and a comb teeth number of 23 and a comb spacing of $5 \mathrm{GHz}$ and a comb teeth number of 21 are demonstrated in the experiment.

\section{OPERATION PRINCIPLE}

The evolution process of the proposed three-stage OFC generator is shown in Figure 1. $f_{m}$ and $f_{s}$ are the working frequencies of the master laser (ML) and the free-running slave laser (SL), as shown in Figure 1A. The SL that is optically injected by the ML can be piloted to enter the period-one oscillation state under appropriate injection conditions, and a primary seed OFC with a comb spacing of $\Delta f\left(\Delta f=f_{m}-f_{s}\right)$ is generated in the first stage, which has sparse comb teeth, as shown in Figure 1B. This primary seed OFC is then sent into a phase modulator (PM) and modulated by the external RF signal at the frequencies of subharmonics of the oscillation period. The primary seed OFC teeth are locked by the subharmonic electro-optic modulation sidebands in the second stage, which will fix the phase relationship of the comb teeth through electro-optic modulation. Interestingly, similar to the dynamic process that is the optical injection locking being able to occur in a certain range of the detuning frequency between the ML and the SL, this 

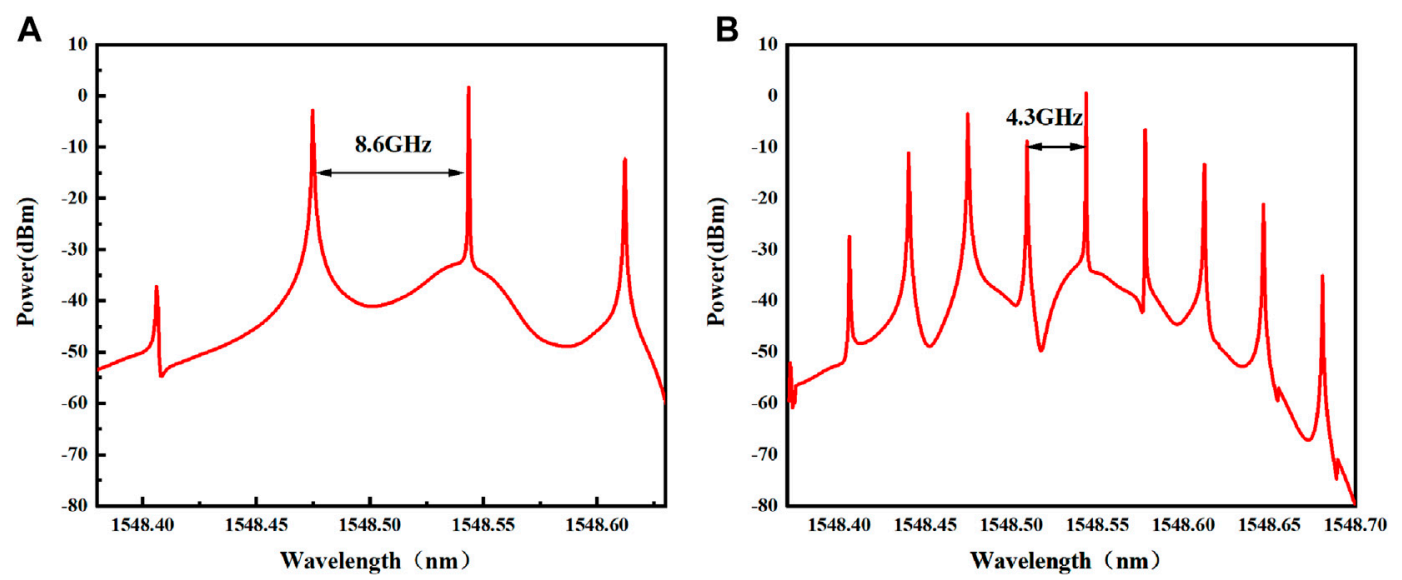

FIGURE 2 | (A) Primary seed OFC. (B) Subharmonic-locked OFC obtained by simulation.

subharmonic locking behavior stays robust in a certain frequency deviation between the period-one frequency and the subharmonic frequency. In addition, the number of comb teeth of this subharmonic-locked OFC increases, and the comb spacing is now $\Delta f / N$ (at $1 / N$ subharmonic electro-optic modulation, $N=1,2,3 \ldots$ ), as shown in Figure $\mathbf{1 C}$ at $1 / 2$ subharmonic electro-optic modulation. Then the subharmoniclocked OFC with the locked phase relationship between the comb teeth is sent into a semiconductor optical amplifier (SOA).

In the SOA, if the comb teeth at frequencies of $f_{m}-1.5 \Delta f, f_{m}-$ $2 \Delta f, f_{m}-2.5 \Delta f$ satisfy the phase matching condition, they will interact with each other and bring out the FWM effect, which leads to the parametric amplification of the frequency mode $f_{m}-1.5 \Delta f$ while generating the signal light with the frequency of $f_{m}-3 \Delta f$. The generated signal light with the two comb teeth at frequencies of $f_{m}-2 \Delta f$ and $f_{m}-2.5 \Delta f$ will continue to bring out the FWM effect under the condition of phase matching, which will lead to another new signal light at the frequency of $f_{m}-3.5 \Delta f$ being generated and new parametric amplification being carried out at the same time. The comb teeth at frequencies of $f_{m}-$ $3 \Delta f, f_{m}-3.5 \Delta f \ldots f_{m}-n \Delta f$ will be generated in turn. Similarly, the comb teeth at frequencies of $f_{m}+2 \Delta f, f_{m}+2.5 \Delta f \ldots f_{m}+n \Delta f$ will be generated at the side of high frequency, and the intensity of the newly generated modes will be increased in these parametric amplification processes. Consequently, the number of comb teeth becomes greater in the third stage, and the newly generated OFC becomes flatter, namely, the FWM-broadened OFC, as shown in Figure 1D. In summary, the robust OFC is obtained after experiencing a three-stage evolutionary process, including the period-one oscillation state in the optically injected laser, the electro-optic modulation in the PM, and the FWM effect in the SOA.

\section{SIMULATION RESULTS}

To verify the proposed scheme, simulation is conducted using Optisystem. When the detuning frequency is set to $5 \mathrm{GHz}$ and the injected intensity is $-17.5 \mathrm{dBm}$, the SL enters the period-one oscillation state and the primary seed OFC is generated, as shown in Figure 2A. Due to the redshift of $3.6 \mathrm{GHz}$ caused by optical injection, the frequency spacing between the comb teeth is 8.6 GHz. The primary seed OFC has few comb teeth, only 4, and is not flat in intensity. In order to increase the number of comb teeth and stabilize the phase relationship between the comb teeth, a PM is used to modulate the seed OFC, and an RF signal is employed, whose frequency is set to $4.3 \mathrm{GHz}$, which is half of the oscillation period of $8.6 \mathrm{GHz}$. For the subharmonic-locked OFC, the number of comb teeth is increased to 10 , and the comb spacing is equal to the frequency of the modulation RF signal, as shown in Figure 2B.

The subharmonic-locked OFC is then sent into the following SOA, and the FWM effect in the SOA is induced to relay the increasing of the number of comb teeth. The corresponding simulation results are shown in Figure 3. It can be seen that the FWM effect drives the number of comb teeth increasing in an obvious manner, and the number of comb teeth increases with the increase in the injection current of the SOA. Compared with the primary seed OFC, the number of comb teeth is up to 35 when the injection current of the SOA is $200 \mathrm{~mA}, 50$ when it is $400 \mathrm{~mA}$, 65 when it is $600 \mathrm{~mA}, 79$ when it is $800 \mathrm{~mA}$, and 101 when it is $1000 \mathrm{~mA}$. The cascaded subharmonic electro-optic modulation and the relaying FWM effect can effectively improve the primary seed OFC in terms of the number and flatness of its comb teeth.

\section{EXPERIMENT RESULTS AND DISCUSSION}

Experiments based on the scheme in Figure 4 are performed to verify the proposed scheme and the simulation results above. The light emitted by the ML passes through the optical isolator (OI), the variable optical attenuator (VOA), and the polarization controller (PC1) in sequence and is then sent into the 3-port optical circulator (OC) at the first port. The SL is connected to the second port of this OC. Due to the perturbation by the external coherence light from the ML, the SL can be controlled into the 


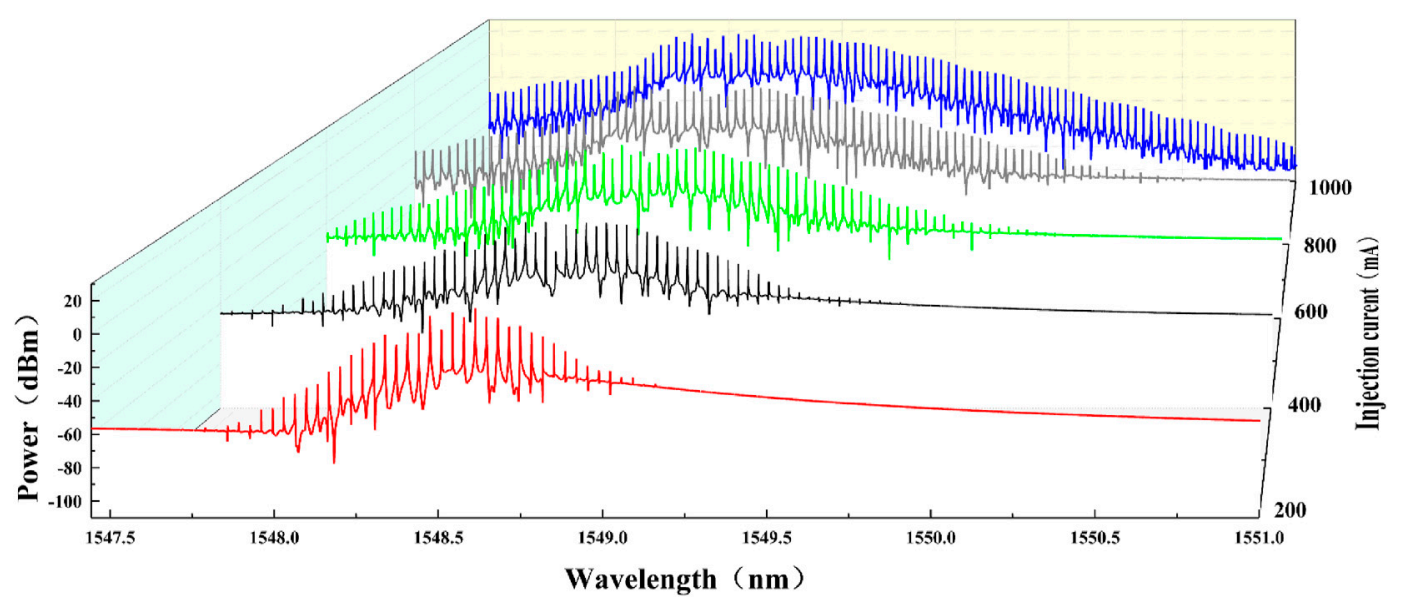

FIGURE 3 | OFCs obtained by simulation under different injection currents of the SOA

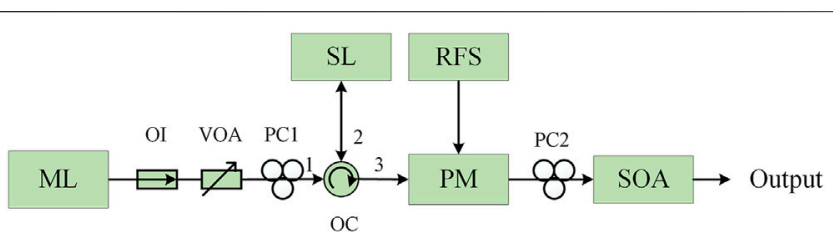

FIGURE 4 | Experimental setup of the proposed system. ML, master laser; SL, slave laser; RFS, RF source; PM, phase modulator; SOA, semiconductor optical amplifier; OI, optical isolator; VOA, variable optical attenuator; PC, polarization controller.

period-one oscillation state, and the primary seed OFC is experimentally generated, which is directly sent into the PM at the third port of the OC. After being modulated in the PM, it is sent into the SOA through the PC2. Finally, the enhanced OFC can be obtained at the output end of the SOA. As analyzed above, the reason for modulating the primary seed OFC from the SL is that the phase stability between the frequency modes of the period-one oscillation state is poor. Such a primary seed OFC is unstable in phase due to carriers in the resonance cavity of the SL. With the use of subharmonic electro-optic modulation, the phase relationship between the comb teeth can be locked and stabilized in the process of the electro-optic modulation, and the original comb teeth are locked to the modulation sidebands at the same frequency. At the same time, subharmonic electro-optic modulation can also make the primary seed OFC teeth become more in number. The function of the PC2 is to control the polarization state of the subharmonic-locked seed OFC because the FWM effect is polarization dependent. By adjusting the PC, the FWM efficiency in the SOA can be maximized.

The bias current of the SL is set to $19.72 \mathrm{~mA}$, the temperature of the laser control system is set to $19.81^{\circ} \mathrm{C}$, the working wavelength is $1554.1460 \mathrm{~nm}$, and the output power is $-3 \mathrm{dBm}$ for the SL. Adjusting the injection intensity by the VOA and the working wavelength of the ML (i.e., changing the detuning frequency between the ML and the SL), the primary seed OFC with flexible and adjustable comb spacing can be obtained. When the detuning frequency is $6 \mathrm{GHz}$, a primary seed OFC with a comb spacing of $8 \mathrm{GHz}$ and a comb teeth number of eight is generated, as shown in Figure 5A. The reason why the comb spacing is larger than the detuning frequency between the ML and the SL is the redshift of the cavity resonant mode wavelength of the SL due to optical injection. It can be seen that the period-one oscillation state can naturally provide a primary seed OFC with tunable comb spacing, but the number of comb teeth is relatively small and its flatness is poor. When the primary seed OFC is modulated by $1 / 2$ subharmonic at $18 \mathrm{dBm}$, the comb spacing becomes $4 \mathrm{GHz}$ and the number of comb teeth becomes 16, as shown in Figure 5B. The comb spacing of the OFC modulated by the $1 / 2$ subharmonic is halved, the number of comb teeth is doubled, and the envelope of comb teeth becomes flatter as well.

The subharmonic-locked OFC with a comb spacing of $4 \mathrm{GHz}$ is sent into the following SOA. The performance of the experimentally obtained OFCs varies with the injection current of the SOA, as shown in Figure 6. Because the phase relationship between the comb teeth is stabilized by subharmonic electro-optic modulation, it is observed that the obtained OFC is very stable when the FWM effect occurs in the SOA. With the increase in the injection current of the SOA, the FWM efficiency increases, which leads to the number of comb teeth increasing in an obvious manner, as expected by the simulation.

For the proposed system, when the intensity of the modulation signal is set to $18 \mathrm{dBm}$ and the injection current of the SOA is set to $300 \mathrm{~mA}$, the quality of the primary seed OFC is obviously improved, and we finally get the FWM-broadened OFC with a comb teeth number of 23 and a comb spacing of $4 \mathrm{GHz}$, as shown in Figure 7A. The system can also generate OFCs with different comb spacing. By adjusting the injection parameters, we obtain a primary seed OFC with a comb spacing of $10 \mathrm{GHz}$ and modulate it with the use of the $1 / 2$ subharmonic before sending it into the SOA with an injection current of $300 \mathrm{~mA}$, and then another OFC with a comb spacing of $5 \mathrm{GHz}$ and a comb teeth number of 21 is obtained, as shown in Figure 7B. While in the SOA, the smaller the comb spacing, the higher the FWM efficiency. Moreover, the higher the injection current of the SOA, the higher the FWM efficiency. Here, the authors would like to mention that the injection current of the 

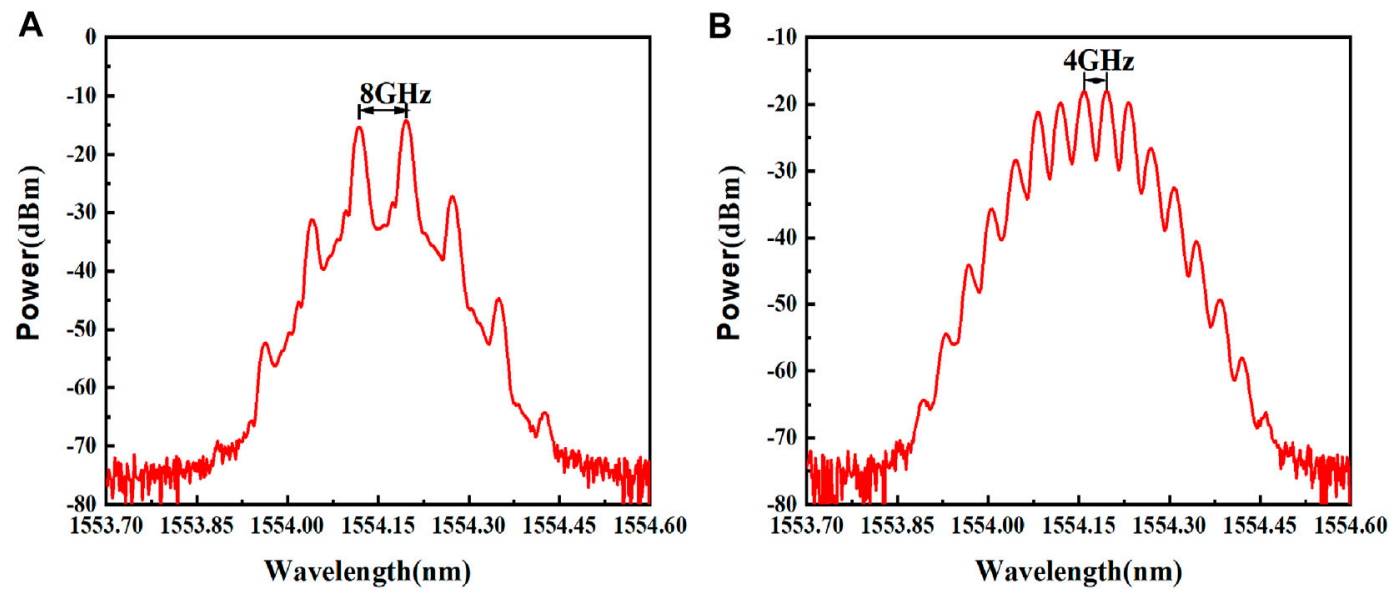

FIGURE 5 | (A) Primary seed OFC. (B) Subharmonic-locked OFC generated in the experiment.

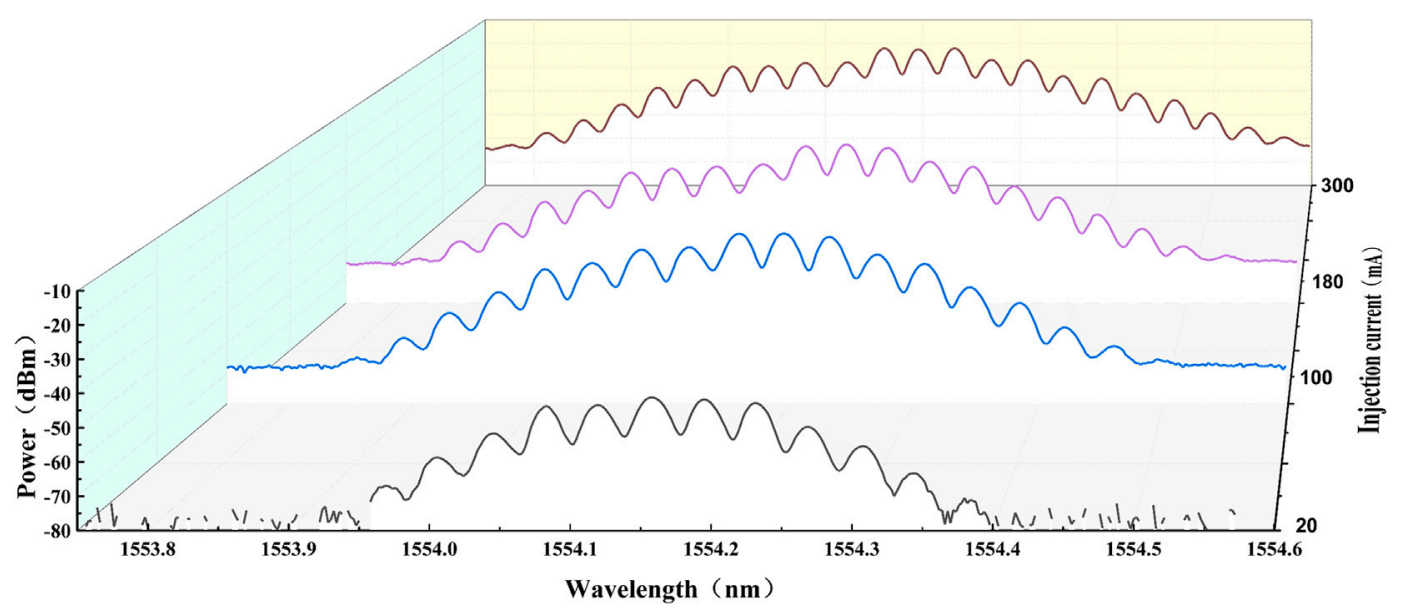

FIGURE 6 | Experimentally obtained FWM-broadened OFCs under different injection currents of the SOA.
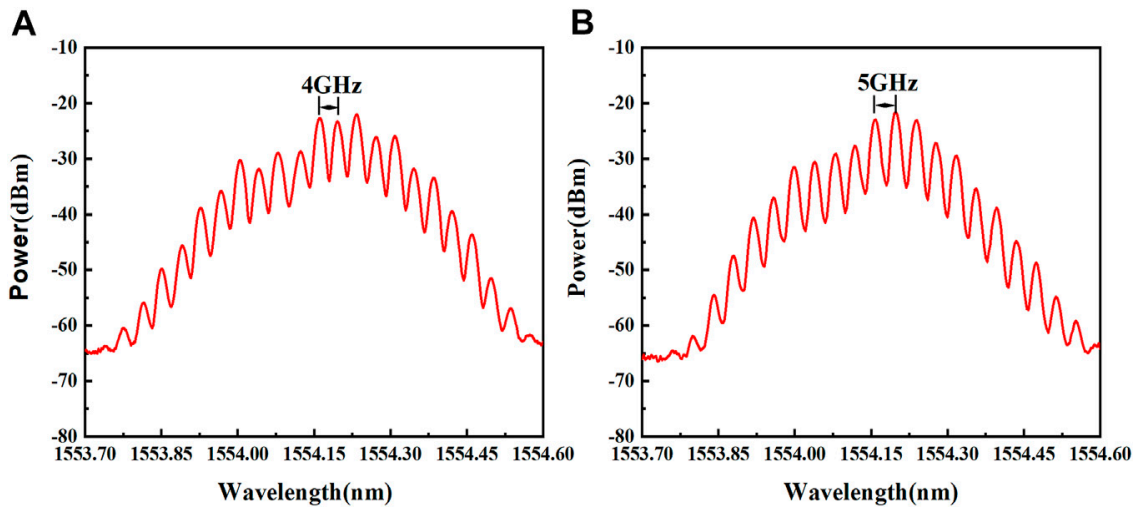

FIGURE 7 | OFCs from the SOA with a comb spacing of (A) $4 \mathrm{GHz}$ and (B) $5 \mathrm{GHz}$. 
SOA in our laboratory is limited to less than $300 \mathrm{~mA}$, which greatly limits the performance enhancement for the proposed OFC.

\section{CONCLUSIONS}

In this article, we have proposed and demonstrated a novel scheme to produce robust OFCs using a three-stage generator, which is based on optical injection and improved by cascaded subharmonic electrooptic modulation and the FWM effect. The obtained OFC features high stability, thanks to the stable phase relationship between the primary seed OFC teeth and the subharmonic electro-optic modulation sidebands through the subharmonic locking. Moreover, it can be flexibly tuned by adjusting the injection parameters of the slaver laser. Finally, robust OFCs with a comb spacing of $4 \mathrm{GHz}$ and a comb teeth number of 23 and a comb spacing of $5 \mathrm{GHz}$ and a comb teeth number of 21 are experimentally obtained and demonstrated. If the injection current of the SOA in the experiment can be higher than $300 \mathrm{~mA}$, the maximum value in our laboratory, the proposed OFC generator can be expected to behave like the simulation situation.

\section{REFERENCES}

1. Altmann RK, Dreissen LS, Salumbides EJ, Ubachs W, and Eikema KSE. Deep-ultraviolet Frequency Metrology of H2 for Tests of Molecular Quantum Theory. Phys Rev Lett (2018) 120(4):1-6. doi:10.1103/ physrevlett.120.043204

2. Biesheuvel J, Karr JP, Hilico L, Eikema KSE, Ubachs WG, and Koelemeij JCJ. Probing QED and Fundamental Constants through Laser Spectroscopy of Vibrational Transitions in HD. Nat Commun (2016) 7(1):10385. doi:10.1038/ ncomms10385

3. Webb JK, King JA, Murphy MT, Flambaum VV, Carswell RF, and Bainbridge MB. Indications of a Spatial Variation of the fine Structure Constant. Phys Rev Lett (2011) 107(19):191101. doi:10.1103/physrevlett.107.191101

4. Huntemann N, Lipphardt B, Tamm C, Gerginov V, Weyers S, and Peik E. Improved Limit on a Temporal Variation of $\mathrm{Mp} / \mathrm{me}$ from Comparisons of $\mathrm{Yb}+$ and Cs Atomic Clocks. Phys Rev Lett (2014) 113(21):210802. doi:10.1103/ physrevlett.113.210802

5. Godun RM, Nisbet-Jones PBR, Jones JM, King SA, Johnson LAM, Margolis HS, et al. Frequency Ratio of Two Optical Clock Transitions in $171 \mathrm{Yb}+$ and Constraints on the Time Variation of Fundamental Constants. Phys Rev Lett (2014) 113(21):210801. doi:10.1103/physrevlett.113.210801

6. Jones DJ, Diddams SA, Ranka JK, Stentz AS, Windeler RS, Hall JL, et al. Carrier-envelope Phase Control of Femtosecond Mode-Locked Lasers and Direct Optical Frequency Synthesis. Science (2000) 288(5466):635-9. doi:10.1126/science.288.5466.635

7. Yu JJ, Dong Z, Zhang JW, Xiao X, Chien H-C, and Chi N. Generation of Coherent and Frequency-Locked Multi-Carriers Using Cascaded Phase Modulators for $10 \mathrm{~Tb} / \mathrm{s}$ Optical Transmission System. J Lightwave Technol (2012) 30(4):458-65. doi:10.1109/jlt.2011.2173460

8. Coddington I, Swann WC, and Newbury NR. Coherent Dual-Comb Spectroscopy at High Signal-To-Noise Ratio. Phys Rev Lett (2010) 82(4): 043817. doi:10.1103/physreva.82.043817

9. Zhang FZ, Wu J, Li Y, and Lin JT. Flat Optical Frequency Comb Generation and its Application for Optical Waveform Generation. Opt Commun (2013) 290(1):37-42. doi:10.1016/j.optcom.2012.10.051

10. Chae E, Nakashima K, Ikeda T, Sugiyama K, and Yoshioka K. Direct PhaseLocking of a Ti:Sapphire Optical Frequency Comb to a Remote Optical Frequency Standard. Opt Express (2019) 27(11):15649-61. doi:10.1364/ oe.27.015649

\section{DATA AVAILABILITY STATEMENT}

The raw data supporting the conclusions of this article will be made available by the authors, without undue reservation.

\section{AUTHOR CONTRIBUTIONS}

SL and $Y Z$ designed the research and discussed it with $\mathrm{ZZ}, \mathrm{SZ}$, and YL. SL, KY, and GW completed the experiment together. ZZ, SZ, and YL participated in the analysis and discussion of the experimental results. SL drafted the content of the manuscript. $\mathrm{YZ}$ was the corresponding author and guided and revised the manuscript.

\section{FUNDING}

This work was supported by the National Key R \& D Program of China (2018YFE0201900) and the National Natural Science Foundation of China (NSFC) (61927821).

11. Davila-Rodriguez J, Bagnell K, and Delfyett PJ. Frequency Stability of a $10 \mathrm{GHz}$ Optical Frequency Comb from a Semiconductor-Based Mode-Locked Laser with an Intracavity 10,000 Finesse Etalon. Opt Lett (2013) 38(18):3665-8. doi:10.1364/OL.38.003665

12. Hellwig T, Rieger S, and Fallnich C. An All-Optically Stabilized Frequency Comb Based on a Mode-Locked Fiber Laser. Opt Lett (2014) 39(3):525-7. doi:10.1364/OL.39.000525

13. Li JP, Zhang XG, and Xi LX. Generation of Stable and High-Quality FrequencyLocked Carriers Based on Improved Re-circulating Frequency Shifter. Opt Commun (2012) 285(20):4072-5. doi:10.1016/j.optcom.2012.06.014

14. Li JP, and Li ZH. Frequency-locked Multicarrier Generator Based on a Complementary Frequency Shifter with Double Recirculating FrequencyShifting Loops. Opt Lett (2013) 38(3):359-61. doi:10.1364/ol.38.000359

15. Wang Q, Huo L, Xing YF, and Zhou BK. Ultra-flat Optical Frequency Comb Generator Using a Single-Driven Dual-Parallel Mach-Zehnder Modulator. Opt Lett (2014) 39(10):3050-3. doi:10.1364/ol.39.003050

16. Qu K, Zhao SH, Li X, Zhu ZH, Liang D, and Liang DY. Ultra-flat and Broadband Optical Frequency Comb Generator via a Single MachZehnder Modulator. IEEE Photon Technol Lett (2017) 29(2):255-8. doi:10.1109/lpt.2016.2640276

17. Dai J, Xu XY, Wu ZL, Dai YT, Yin FF, Zhou Y, et al. Self-oscillating Optical Frequency Comb Generator Based on an Optoelectronic Oscillator Employing Cascaded Modulators. Opt Express (2015) 23(23):30014-9. doi:10.1364/ oe. 23.030014

18. Plascak ME, Bustos Ramirez R, Bagnell K, and Delfyett PJ. Tunable Broadband Electro-Optic Comb Generation Using an Optically Filtered Optoelectronic Oscillator. Photon Technol Lett (2018) 30(4):335-8. doi:10.1109/lpt.2017.2788361

19. Yang X, Richardson DJ, and Petropoulos P. Broadband, Flat Frequency Comb Generated Using Pulse Shaping-Assisted Nonlinear Spectral Broadening. IEEE Photon Technol Lett (2013) 25(6):543-5. doi:10.1109/lpt.2013.2242882

20. Yu SY, Bao FD, and $\mathrm{Hu} \mathrm{H}$. Broadband Optical Frequency Comb Generation with Flexible Frequency Spacing and center Wavelength. IEEE Photon J. (2018) 10(2):1-7. doi:10.1109/jphot.2018.2832842

21. Ge MF, Zhang YL, Xia Y, Zhang ZY, Zhang SJ, and Liu Y. Optical-injectionseeded Optical Frequency Comb Generation Promoted by the Sub-harmonic Modulation. In: The 18th International Conference on Optical Communications and Networks; 2019 Aug 05-08; Huangshan, China (2019). doi:10.1109/icocn.2019.8933971

22. Zhang YL, Ge MF, Zhang ZY, Zhang SJ, and Liu Y. Self-recirculating Modulation-Enhanced Optical Frequency Comb Generation Pumped by 
Optical Injection. In: SPIE/COS Photonics Asia; 2019 Oct 20-23; Hangzhou, China (2019). doi:10.1117/12.2538935

Conflict of Interest: The authors declare that the research was conducted in the absence of any commercial or financial relationships that could be construed as a potential conflict of interest.

Publisher's Note: All claims expressed in this article are solely those of the authors and do not necessarily represent those of their affiliated organizations, or those of the publisher, the editors and the reviewers. Any product that may be evaluated in this article, or claim that may be made by its manufacturer, is not guaranteed or endorsed by the publisher.

Copyright $\odot 2021$ Zhang, Liao, Wang, Yang, Zhang, Zhang and Liu. This is an openaccess article distributed under the terms of the Creative Commons Attribution License (CC BY). The use, distribution or reproduction in other forums is permitted, provided the original author(s) and the copyright owner(s) are credited and that the original publication in this journal is cited, in accordance with accepted academic practice. No use, distribution or reproduction is permitted which does not comply with these terms. 